\title{
A 68 years Old Man with Compressive Chest Pain and Breathlessness
}

\author{
FACADER $^{\mathrm{a}}, \mathrm{MM} \mathrm{HAQ}^{\mathrm{b}}$, N GHAFOOR ${ }^{\mathrm{c}}$
}

(J Banagladesh Coll Phys Surg 2015; 33: 232-234)

A 65 year old Bangladeshi male presented with compressive chest pain and respiratory distress for 2 days. He had hoarseness of voice and breathlessness for 1 year. He was hypertensive, diabetic and dyslipidaemic, and had a past history of ischaemic.

Physical examination revealed a pulse rate of $92 / \mathrm{min}$, blood pressure (BP) of 130/100mmHg, unremarkable praeordial and lung auscultation. ECG showed right bundle branch block. Troponin I and NTPro-BNP were normal. Echocardiogram revealed concentric LV hypertrophy with normal LV systolic dysfunction, and grade I diastolic dysfunction. Chest $\mathrm{X}$ ray showed widened mediastinum with prominent aortic knob (Fig.-1).

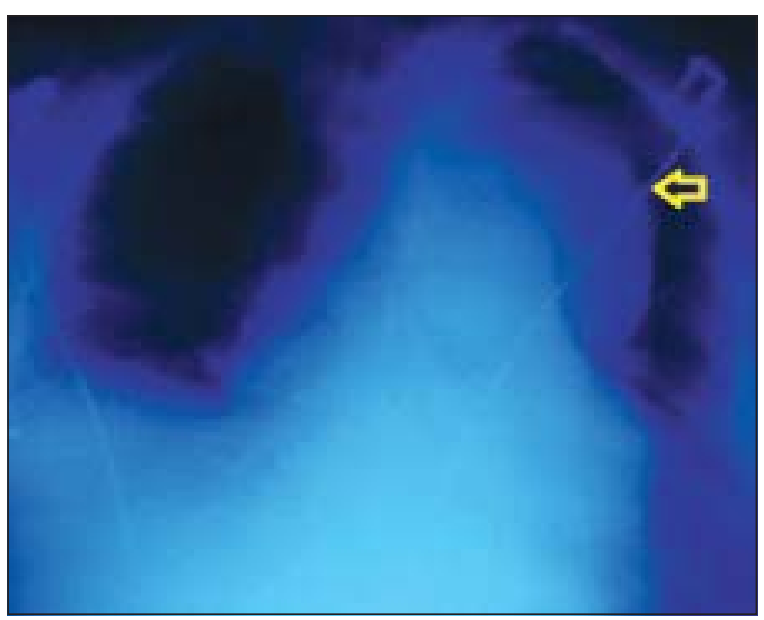

Fig.-1: Chest $X$ ray AP view showing widened mediastinum with prominent aortic knob.

a. Dr. F. Aaysha Cader, Department of Cardiology, Ibrahim Cardiac Hospital \& Research Institute, Dhaka.

b. Prof. M. Maksumul Haq, Department of Cardiology, Ibrahim Cardiac Hospital \& Research Institute, Dhaka.

c. Nusrat Ghafoor, Department of Radiology \& Imaging, Ibrahim Cardiac Hospital \& Research Institute, Dhaka.

Address of Correspondence: Dr. F. Aaysha Cader, Department of Cardiology, Ibrahim Cardiac Hospital \& Research Institute, Dhaka. Email: aaysha.cader@gmail.com
CT aortography showed a large saccular dilatation of the arch of aorta (Fig.-2) distal to the left subclavian artery measuring $8.3 \mathrm{~cm}$ transversely with mural thrombus. Another focal fusiform dilatation of proximal thoracic aorta measuring about $6.9 \mathrm{~cm}$ at maximum was seen, with eccentric mural thrombus leaving a patent lumen of $\sim 2.7 \mathrm{~cm}$. Fusiform diataion was also seen in the abdominal aorta (Fig.-3). No dissection seen. Extensive workups for thrombophilia were negative.

He was given beta blockers and ACE inhibitor for BP control, aspirin, and statin, in line with ACCF/AHA recommendations ${ }^{1}$. Given the mural thrombus, he was treated with enoxaparin, and discharged on warfarin. He was offered thoracic endovascular repair of aneurysm (TEVAR) or open surgical repair of thoracic aortic aneurysm (TAA).

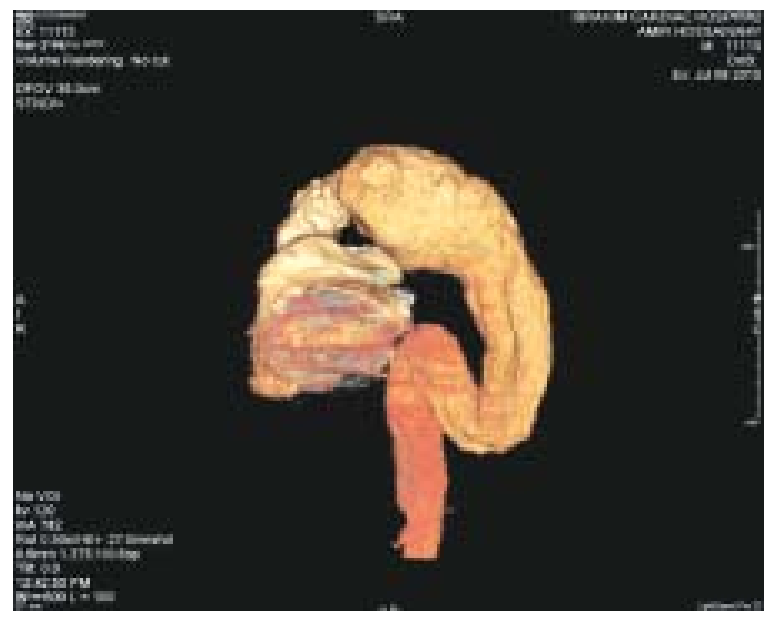

Fig.-2: CT aortography image showing large saccular dilatation of the arch of aorta and focal fusiform dilatations of descending thoracic and abdominal aortae. 


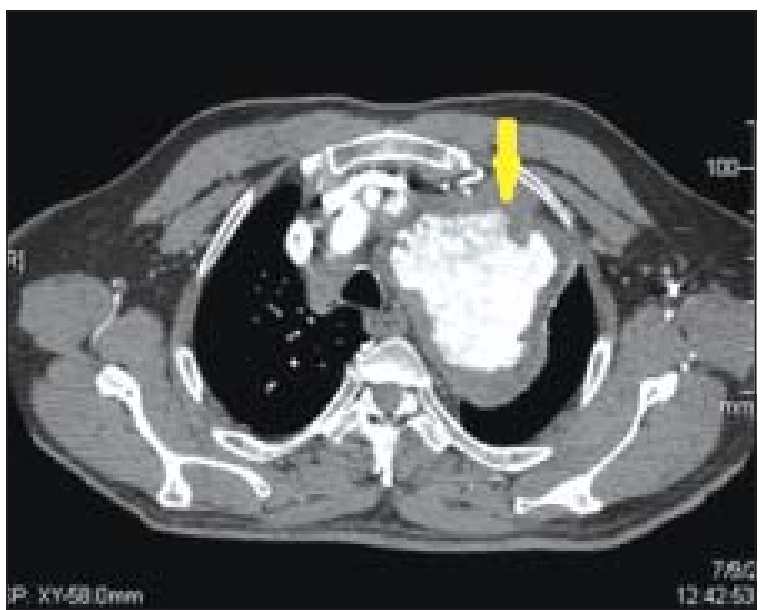

(a)

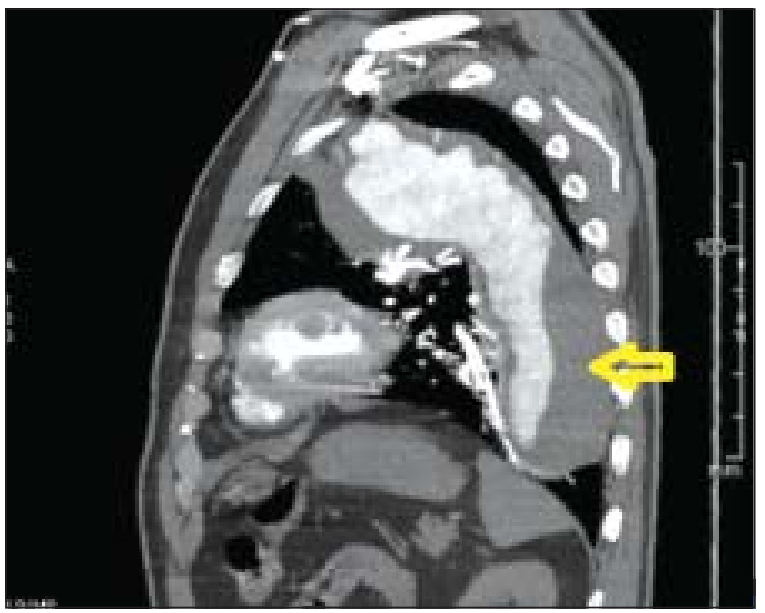

(c)

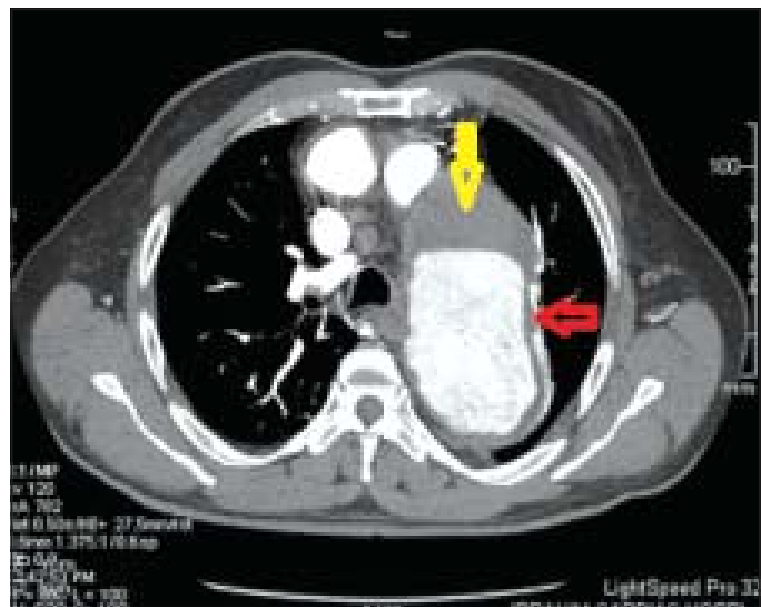

(b)

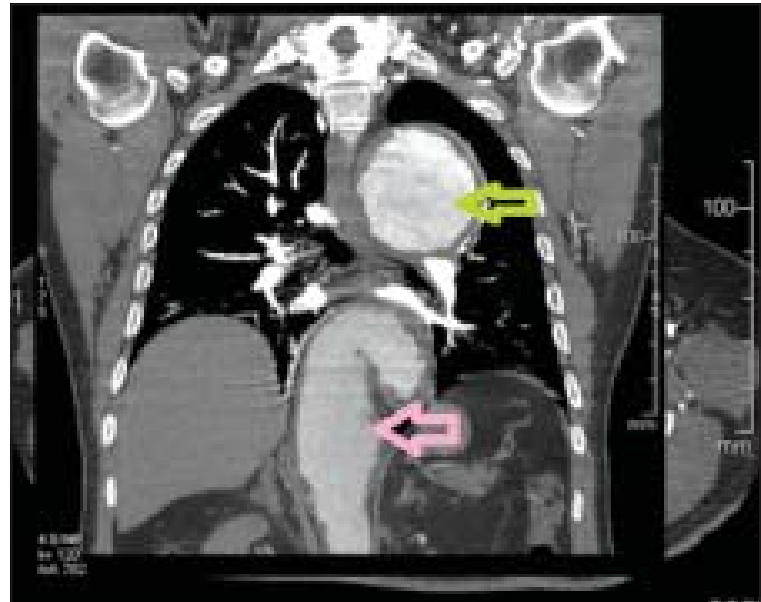

(d)

Fig.-3: CT scan of chest (a) : Axial plane: Ascending aortic aneurysm showing filling defect (yellow arrow) denoting thrombus. (b): Axial plane: Descending TAA (red arrow) with thrombus (yellow arrow). (c): sagittal plane: descending TAA showing thrombus (yellow arrow). (d): coronal plane showing ascending aortic aneurysm (green arrow) and descending TAA and abdominal aortic aneurysms (pink arrow).

\section{Discussion:}

An aortic aneurysm is diagnosed when the ascending aorta is larger than $5 \mathrm{~cm}$ and the descending aorta is larger than $4 \mathrm{~cm}^{2}$. Aortic root or ascending aortic aneurysms ( $\sim 60 \%$ ) are the most common TAA, followed by descending aorta (35-40 \%) and aortic arch (<10\%) 2,3 . Thoracoabdominal aneurysms constitute approximately $3 \%$ of all aortic aneurysms and are usually diffuse and atherosclerotic in nature ${ }^{3}$. Atherosclerosis is the overall most common cause of aneurysm, accounting for $70 \%{ }^{2}$. In contrast to the ascending aorta, the majority of descending TAAs are atherosclerotic ${ }^{2,3}$.
Most of them present in the sixth and seventh decades of life, with a male predominance, and involvement of abdominal aorta in one-third of patients ${ }^{2}$.

The most likely aetiology in this case was atherosclerosis, as for most descending TAA, compounded by risk factors of smoking, hypertension, and older age ${ }^{4}$. Descending TAAs are typically fusiform, often begin distal to the origin of the left subclavian artery $^{3}$ and coexist with abdominal or arch aneurysms ${ }^{3}$. Most TAA and abdominal aortic aneurysms are clinically silent, with the aneurysm discovered incidentally on 
chest radiography, echocardiography, CT or $\mathrm{MRI}^{4}$. Alternatively, rupture may constitute the first manifestation $^{3}$. 5\%-10\% of patients experience symptoms, such as chest or back pain. Aortic arch or descending TAAs may also produce hoarseness of voice ("dysphagia lusoria") from compression of the recurrent laryngeal nerve ${ }^{3}$.

Atherosclerotic aneurysms are commonly associated with mural thrombosis, posing the additional risk of systemic embolization, causing occlusion of distal vessels $^{3-6}$. Other serious complications of TAA are dissection and rupture.

Most TAAs are evident on chest radiographs ${ }^{3,4}$ but chest $\mathrm{X}$ rays cannot exclude their diagnosis. Trans thoracic echocardiography may not thoroughly characterise arch and descending TAA. Transoesophageal echocardiography can usually image most of the aorta ${ }^{4}$. However, the best imaging modality for TAA is Computed tomography (CT) or magnetic Resonance Imaging (MRI). It has several advantages, including rapid scan times, wider availability and the ability to image the three-dimensional structure of the aorta along its entire course $\mathrm{e}^{1-3}$. Furthermore CT angiography and contrast-enhanced MRI are highly accurate in the evaluation and follow up of patients undergoing endovascular TAA therapy, and are also preferred over aortography in most cases ${ }^{3}$.

The optimal management of TAA mural thrombi has not been clearly established and is influenced by the localisation of the thrombus and patient co-morbidities. Therapeutic strategies include anticoagulation, thrombolysis, interventional modalities such as thromboaspiration, or balloon-catheter thrombectomy, and open surgical procedures such as thrombectomy, thromboendarterectomy, and aortic prosthetic replacement ${ }^{7}$. Strict control of hypertension (target goal $<130 / 80 \mathrm{mmHg}$ in diabetics), optimization of lipid profile with statins (target LDL $<70 \mathrm{mg} / \mathrm{dL}$ ), smoking cessation and glycaemic control should be instituted. Betablockers and ACE inhibitors or Angiotensin receptor blockers are recommended anti-hypertensive choices (class IIa) ${ }^{1}$.

Intervention is recommended at larger diameters for aneurysms of the descending aorta ${ }^{1}$. Symptomatic aortic aneurysms at any level should be resected regardless of size. Modalities include open surgical repair (OSR) or Thoracic Endovascular aneurysm repair (TEVAR). TEVAR is a far less invasive alternative to OSR of descending TAAs, with lower morbidity and mortality rates, provided the aortic anatomy has adequate landing zones to accommodate the endograft ${ }^{1,8}$. Long term surveillance of the aorta with imaging is imperative, with re-evaulation at 6 months after discovery of the aneurysm to document its stability. For degenerative TAAs bi-annual imaging is recommended for aneurysms between 4.5 to $5.4 \mathrm{~cm}$, and annual if $3.5-4.4 \mathrm{~cm}^{4}$.

\section{References:}

1. Hiratzka LF, Bakris GL, Beckman JA, Bersin RM, Carr VF, Casey DE Jr,et al. 2010 ACCF/ AHA/ AATS/ ACR/ ASA/ SCA/ SCAI/ SIR/ STS/ SVM guidelines for the diagnosis and management of patients with Thoracic Aortic Disease. Circulation. 2010;121(13):e266-369. doi: 10.1161/CIR.0b013e3181d4739e. Epub 2010 Mar 16.

2. Rajiah P. CT and MRI in the Evaluation of Thoracic Aortic Diseases. Int J Vasc Med. 2013;2013:797189. doi: 10.1155/2013/797189. Epub 2013 Dec 11.

3. Elefteriades JA, Olin JW, Halperin JL. Diseases of the Aorta. In: Fuster V, Walsh RA, Harrington RA, editors. Hurst's The Heart. New York: The McGraw-Hill Companies, 2011, p2261-89

4. Braverman AC. Diseases of the Aorta. In : Mann DL, Zipes DP, Libby P, Bonow RO, editors. Braunwald's Heart Disease: A Textbook of Cardiovascular Medicine. 10th ed. Philadelphia PA: Elsevier Saunders; 2015, p 1277-1311.

5. Ungprasert P, Ratanapo S, Cheungpasitporn W. Management in Thoracic Aorta Mural Thrombi: Evidence Based Medicine and Controversy. Emerg Med (Los Angel) 1:e104. doi: 10.4172/2165-7548.1000e104.

6. Reber PU, Patel AG, Stauffer E, Müller MF, Do DD, Kniemeyer HW. Mural aortic thrombi: An important cause of peripheral embolization. J Vasc Surg. 1999;30(6):1084-9.

7. Piffaretti G, Tozzi M, Mariscalco G, Bacuzzi A, Lomazzi C, Rivolta $\mathrm{N}$, et al. Mobile thrombus of the thoracic aorta: management and treatment review. Vasc Endovascular Surg. 2008;42(5):405-11.

8. Adams JD, Garcia LM, Kern JA. Endovascular repair of the thoracic aorta. Surg Clin North Am. 2009;89(4):895912 . 